\title{
Research Article \\ Coherent and Incoherent Neutral Current Scattering for Supernova Detection
}

\author{
P. C. Divari \\ Department of Physical Sciences and Applications, Hellenic Army Academy, Vari 16673, Attica, Greece \\ Correspondence should be addressed to P. C. Divari, pdivari@gmail.com
}

Received 28 December 2011; Revised 15 March 2012; Accepted 30 March 2012

Academic Editor: Guey-Lin Lin

Copyright (c) 2012 P. C. Divari. This is an open access article distributed under the Creative Commons Attribution License, which permits unrestricted use, distribution, and reproduction in any medium, provided the original work is properly cited.

The total cross sections as well as the neutrino event rates are calculated in the neutral current neutrino scattering off ${ }^{40} \mathrm{Ar}$ and ${ }^{132} \mathrm{Xe}$ isotopes at neutrino energies $\left(E_{v}<100 \mathrm{MeV}\right)$. The individual contribution coming from coherent and incoherent channels is taking into account. An enhancement of the neutral current component is achieved via the coherent $\left(0_{\mathrm{gs}}^{+} \rightarrow 0_{\mathrm{gs}}^{+}\right)$channel which is dominant with respect to incoherent $\left(0_{\mathrm{gs}}^{+} \rightarrow J_{f}\right)$ one. The response of the above isotopes as a supernova neutrino detection has been considered, assuming a two parameter Fermi-Dirac distribution for the supernova neutrino energy spectra. The calculated total cross sections are tested on a gaseous spherical TPC detector dedicated for supernova neutrino detection.

\section{Introduction}

It is well known that neutrinos and their interactions with nuclei have attracted a great deal of attention, since they play a fundamental role in nuclear physics, cosmology, and in various astrophysical processes, especially in the dynamics of core-collapse supernovanucleosynthesis [1-11]. Moreover, neutrinos proved to be interesting tools for testing weak interaction properties, by examining nuclear structure and for exploring the limits of the standard model [12]. In spite of the important role the neutrinos play in many phenomena in nature, numerous questions concerning their properties, oscillation characteristics, their role in star evolutions and in the dark matter of the universe, and so forth remain still unanswered. The main goal of experimental [13-17] and theoretical studies [18-27] is to shed light on the above open problems to which neutrinos are absolutely crucial.

Among the probes which involve neutrinos, the neutrino-nucleus interaction possess a prominent position [28-34]. Thus, the study of neutrino scattering with nuclei is a good way to detect or distinguish neutrinos of different flavor and explore the basic structure of the weak interactions. Also, specific neutrino-induced transitions between discrete nuclear 


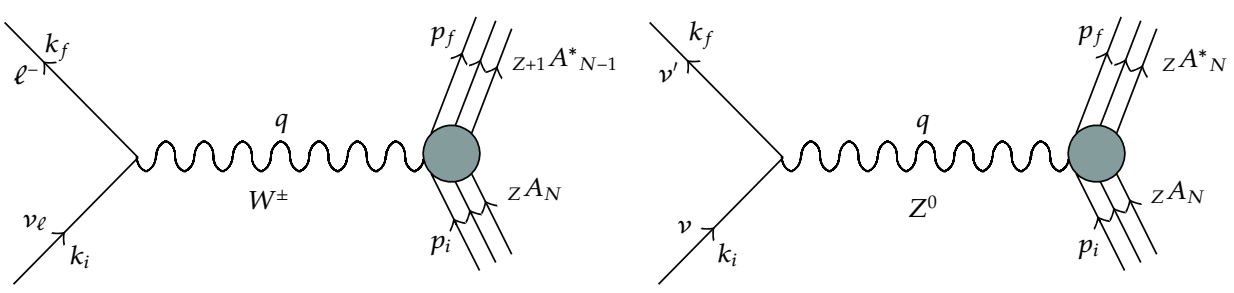

(a)

(b)

Figure 1: Feynman-diagram of lowest order for: (a) the CC neutrino-nucleus reactions $v_{l}+(A, Z) \rightarrow l^{-}+$ $(A, Z+1)^{*}$, and $(\mathrm{b})$ the NC neutrino-nucleus processes $v+(A, Z) \rightarrow v^{\prime}+(A, Z)^{*}$. The diagrams which correspond to the antineutrino reactions are similar.

states with good quantum numbers of spin, isospin, and parity allows us to study the structure of the weak hadronic currents. Furthermore, terrestrial experiments performed to detect astrophysical neutrinos, as well as neutrino-induced nucleosynthesis interpreted through several neutrino-nucleus interaction theories, constitute good sources of explanation for neutrino properties. There are four categories of neutrino-nucleus processes: the two types of charged-current (CC) reactions of neutrinos and antineutrinos and the two types of neutral-current $(\mathrm{NC})$ ones. In the charged-current reactions a neutrino $v_{l}$ (antineutrino $\bar{v}_{l}$ ) with $l=e, \mu, \tau$ transforms one neutron (proton) of a nucleus to a proton (neutron), and a charged lepton $l^{-}$(anti-lepton $l^{+}$) is emitted as

$$
\begin{aligned}
& v_{l}+(A, Z) \longrightarrow l^{-}+(A, Z+1)^{*}, \\
& \bar{v}_{l}+(A, Z) \longrightarrow l^{+}+(A, Z-1)^{*} .
\end{aligned}
$$

These reactions are also called neutrino (antineutrino) capture, since they can be considered as the reverse processes of lepton capture. They are mediated by exchange of heavy $W^{ \pm}$bosons according to the (lowest order) Feynman diagram shown in Figure 1(a). In neutral-current reactions (neutrino scattering) the neutrinos (antineutrinos) interact via the exchange of neutral $Z^{0}$ bosons (see Figure $1(b)$ ) with a nucleus as

$$
\begin{aligned}
& \mathcal{v}+(A, Z) \longrightarrow \mathcal{v}^{\prime}+(A, Z)^{*}, \\
& \bar{v}+(A, Z) \longrightarrow \bar{v}^{\prime}+(A, Z)^{*},
\end{aligned}
$$

where $v(\bar{v})$ denote neutrinos (antineutrinos) of any flavor. The neutrino-nucleus reactions leave the final nucleus mostly in an excited state lying below particle-emission thresholds (semi-inclusive processes) [26]. The transitions to energy levels higher than the particlebound states usually decay by particle emission and, thus, they supply light particles that can cause further nuclear reactions.

When a massive star runs out of its nuclear fuel, it collapses under its own gravity [3538]. As a consequence of this collapse, the density and temperature in its core increase and finally the outer shell of the star explodes, emitting a huge amount of energy. That procedure of violent energy emission in interstellar medium is called supernova (SN) explosion. Most part of this energy is carried in the space by neutrinos of all flavors $\left(v_{e}, v_{\mu}, v_{\tau}, \bar{v}_{e}, \bar{v}_{\mu}, \bar{v}_{\tau}\right)$. Although the energy released by an SN explosion is shared equally between neutrinos of all flavors, their energy spectra differ due to the dependence of neutrinos flavor on their 
interaction with nuclei in the stellar gas. The change in gravitational binding energy between the initial stellar core and the final proton-neutron star is about $3 \times 10^{53} \mathrm{erg}, 99 \%$ of which is carried off by all flavors of neutrinos and antineutrinos in about $10 \mathrm{~s}$. The emission time is much longer than the light-crossing time of the protoneutron star because the neutrinos are trapped and then have to be diffused out, eventually escaping the star having energy distribution spectra which are approximated by the Fermi-Dirac (FD) energy distribution ones. In the canonical model [39-41], $v_{e}$ is emitted with temperature $T \simeq 3.5 \mathrm{MeV}, \bar{v}_{e}$ has $T \simeq 5 \mathrm{MeV}$, and all other flavors $\left(v_{x}\right)$ have temperature $T \simeq 8 \mathrm{MeV}$. The temperatures differ from each other because $\bar{v}_{e}$ and $v_{e}$ have charged-current opacities (in addition to the neutral-current opacities common to all flavors) and because the protoneutron star has more neutrons than protons. The neutrinos $v_{x}(x=\mu, \tau, \bar{\mu}, \bar{\tau})$ do not have sufficient energy to produce corresponding leptons in charged-current reactions and interact only through neutral-current interactions and therefore have a higher average energy than $v_{e}$ and $\bar{v}_{e}$, which interact through charged current as well as neutral current. Since the number of neutrons is larger than the protons, $v_{e}$ loses energy much more than $\bar{v}_{e}$ and the average energy for $\bar{v}_{e}$ is more than $v_{e}$.

Precise theoretical estimates of neutrino-nucleus cross-sections, in low and intermediate neutrino energies, are extremely important in modern neutrino physics [28-34]. In the present work, we have performed realistic calculations for the differential and total cross sections of neutrino elastic (coherent) and inelastic (incoherent) scattering off ${ }^{40} \mathrm{Ar}\left(v, v^{\prime}\right){ }^{40} \mathrm{Ar}^{*}$ and ${ }^{132} \mathrm{Xe}\left(\boldsymbol{v}, v^{\prime}\right){ }^{132} \mathrm{Xe}^{*}$ using the quasi-particle random phase approximation (QRPA). The response of noble gases $\mathrm{Ar}$ and $\mathrm{Xe}$ as a supernova neutrino detection is evaluated assuming a two-parameter FD distribution. Since neutrino energies from SN explosions are expected to be higher than those stemming from the solar neutrino, one needs to consider the contributions from higher multipole states. For this reason, we have considered all the QRPA excited states of ${ }^{40} \mathrm{Ar}$ and ${ }^{132} \mathrm{Xe}$ up to $40 \mathrm{MeV}$, in contrast to previous RPA calculations [42] concerning ${ }^{40} \mathrm{Ar}$, which seems to take only a few excited states known by experiment. Moreover, we have investigated the individual contributions coming from the coherent $\left(0_{\mathrm{gs}}^{+} \rightarrow 0_{\mathrm{gs}}^{+}\right)$and incoherent $\left(0_{\mathrm{gs}}^{+} \rightarrow J_{f}\right)$ channels to total neutrino-nucleus cross sections. We found that the coherent channel is dominant versus the incoherent one.

\section{The Primary Supernova Neutrino Flux}

The neutrino spectrum of a core-collapse supernova is believed to be similar to an FD spectrum, with temperatures in the range $3-8 \mathrm{MeV}$ [41]. The FD energy distribution is given by

$$
f_{i}\left(E_{v}\right)=\frac{N_{2}(\alpha)}{T^{3}} \frac{E_{v}^{2}}{1+e^{E_{v} / T-\alpha}}, \quad i=v_{e}, \bar{v}_{e} \text {, or } v_{x}
$$

where $N_{2}(\alpha)$ is the normalization constant depending on the parameter $\alpha$ given by the relation

$$
N_{k}(\alpha)=\left(\int_{0}^{\infty} \frac{x^{k}}{1+e^{x-\alpha}} d x\right)^{-1}
$$




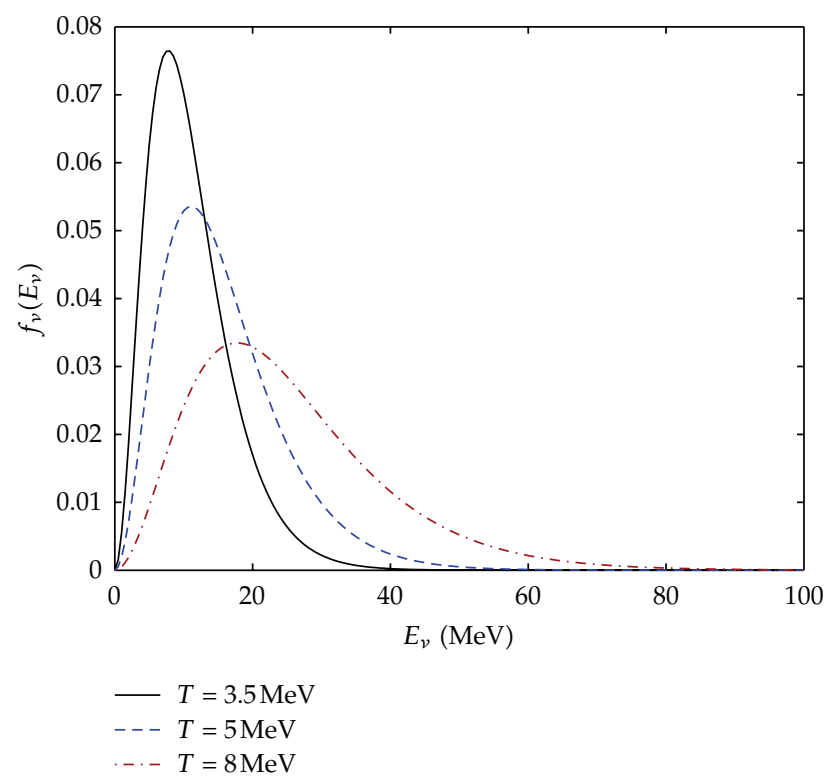

Figure 2: (Color on line). The normalized to unity Fermi-Dirac spectrum for $\alpha=0$.

for $k=2$. Characteristic of the FD energy distribution is that the peak shifts to higher neutrino energies and the width increases as the neutrino energy increases (Figure 2). According to [43], the average neutrino energy $\left\langle E_{v}\right\rangle$ is given by

$$
\left\langle E_{v}\right\rangle=\frac{N_{2}(\alpha)}{N_{3}(\alpha)} T .
$$

Some characteristic values of $\left\langle E_{v}\right\rangle$ are listed in Table 1. Figure 3(a) shows the averaged neutrino energy as a function of the parameter $\alpha$ for various temperatures $T$. As it is seen the introduction of a chemical potential, $\mu=\alpha T$, in the spectrum at fixed neutrino temperature increases the average neutrino energy. From Figure 3(b) it is also seen that at fixed neutrino temperature a nonvanishing chemical potential enhances the averaged neutrino energy.

The interaction of neutrinos with dense neutron rich matter in the core results in the different energy distributions for the various neutrino flavors. The neutrinos $v_{x}(x=\mu, \tau, \bar{\mu}, \bar{\tau})$ do not have sufficient energy to produce corresponding leptons in charged current reactions and interact only through neutral-current interactions and therefore have a higher average energy than $v_{e}$ and $\bar{v}_{e}$, which interact through charged current as well as neutral current. Since the number of neutrons is larger than the protons, $v_{e}$ loses energy much more than $\bar{v}_{e}$ and the average energy for $\bar{v}_{e}$ is more than $v_{e}$. The numerical simulations give the following values of average energy for the different neutrino flavors:

$$
\left\langle E_{v}\right\rangle \sim\left\{\begin{array}{c}
10-11 \mathrm{MeV} v_{e} \\
15-16 \mathrm{MeV} \bar{v}_{e} \\
23-25 \mathrm{MeV} v_{x}, x=\mu, \tau, \bar{\mu}, \bar{\tau}
\end{array}\right\} .
$$


Table 1: The average supernova neutrino energies as a function of the parameters $\alpha$ and T.

\begin{tabular}{lccc}
\hline$\alpha$ & $v_{e}$ & $\left\langle E_{v}\right\rangle(\mathrm{MeV})$ & $\tilde{v}_{e}$ \\
& $T=3.5 \mathrm{MeV}$ & $T=5 \mathrm{MeV}$ & $\boldsymbol{v}_{x}$ \\
& 11.03 & 15.76 & 25.21 \\
0.75 & 11.45 & 16.36 & 26.17 \\
1.50 & 12.08 & 17.26 & 27.61 \\
2.00 & 12.62 & 18.03 & 28.84 \\
3.00 & 13.97 & 19.96 & 31.94 \\
4.00 & 15.63 & 22.33 & 35.73 \\
5.00 & 17.52 & 25.02 & 40.04 \\
\hline
\end{tabular}

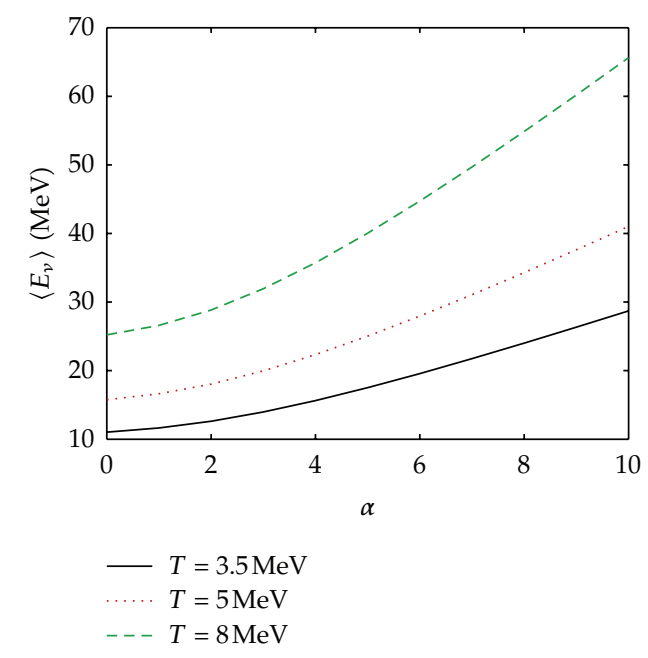

(a)

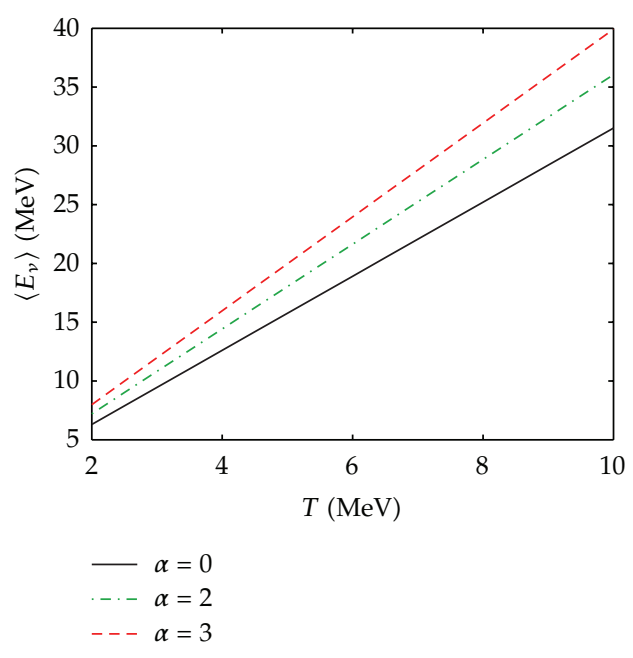

(b)

Figure 3: (Color on line). Averaged neutrino energy as a function of the parameter $\alpha$ for various temperatures $T$ (a). Averaged neutrino energy as a function of temperature for various parameters of chemical potential $\alpha(b)$.

Those average neutrino energies imply that for $\alpha=0$ (3) the values of temperature $T$ are $3.5 \mathrm{MeV}(2.75 \mathrm{MeV})$ for $v_{e}, 5 \mathrm{MeV}(4 \mathrm{MeV})$ for $\bar{v}_{e}$, and $8 \mathrm{MeV}(6 \mathrm{MeV})$ for $v_{x}$.

The number of emitted neutrinos is

$$
N_{v}=\frac{U_{v}}{\left\langle E_{v}\right\rangle}
$$

where $U_{v}=0.5 \times 10^{53} \mathrm{erg}$ per neutrino flavor. Taking the temperature $T$ to be $3.5,5$, and $8 \mathrm{MeV}$ for electron neutrinos $\left(v_{e}\right)$, electron antineutrinos $\left(\bar{v}_{e}\right)$, and all other flavors $\left(v_{x}\right)$ respectively, and the parameter $\alpha$ to be $0 \leq \alpha \leq 5$, then the obtained results for the number of primary neutrinos emitted are shown in Table 2, while the (time averaged) neutrino flux $\Phi_{v}=N_{v} / 4 \pi L^{2}$ at a distance $L=10 \mathrm{Kpc}=3.1 \times 10^{22} \mathrm{~cm}$ is given in Table 3. 
Table 2: The number of primary neutrinos emitted in a typical supernova explosion as a function of the parameters $\alpha$ and $T$ in units of $10^{58}$.

\begin{tabular}{lccc}
\hline$\alpha$ & $v_{e}$ & $N_{v} / 10^{58}$ & \\
$\bar{v}_{e}$ & $T=5 \mathrm{MeV}$ & $\sum_{x} v_{x}$ \\
$T=3.5 \mathrm{MeV}$ & 0.20 & $0.50 \mathrm{MeV}$ \\
\hline 0 & 0.28 & 0.19 & 0.48 \\
0.75 & 0.27 & 0.18 & 0.46 \\
1.50 & 0.26 & 0.17 & 0.44 \\
2.00 & 0.25 & 0.16 & 0.39 \\
3.00 & 0.22 & 0.14 & 0.35 \\
4.00 & 0.20 & 0.12 & 0.32 \\
5.00 & 0.18 &
\end{tabular}

Table 3: The (time integrated) neutrino flux, in units of $10^{12} \mathrm{~cm}^{-2}$, at a distance $10 \mathrm{kpc}$ from the source.

\begin{tabular}{lccc}
\hline$\alpha$ & $v_{e}$ & $\begin{array}{c}\Phi_{v} / 10^{12} \mathrm{~cm}^{-2} \\
\bar{v}_{e} \\
T=5 \mathrm{MeV}\end{array}$ & $\begin{array}{c}\sum_{x} \boldsymbol{v}_{x} \\
T=8 \mathrm{MeV}\end{array}$ \\
\hline 0 & $0.5 \mathrm{MeV}$ & 0.16 & 0.41 \\
0.75 & 0.23 & 0.16 & 0.39 \\
1.50 & 0.23 & 0.15 & 0.37 \\
2.00 & 0.21 & 0.14 & 0.36 \\
3.00 & 0.20 & 0.13 & 0.32 \\
4.00 & 0.18 & 0.12 & 0.29 \\
5.00 & 0.17 & 0.10 & 0.26 \\
\hline
\end{tabular}

\section{Brief Description of the Neutral-Current Neutrino-Nucleus Scattering Formalism}

In the present work we consider neutral-current neutrino-nucleus interactions in which a low or intermediate energy neutrino (or antineutrino) is scattered inelastically from a nucleus $(A, Z)$. The initial nucleus is assumed to be spherically symmetric having ground state a $\left|J^{\pi}\right\rangle=\left|0^{+}\right\rangle$state.

The corresponding standard model effective Hamiltonian in current-current interaction form is written as

$$
\mathscr{\ell}=\frac{G}{\sqrt{2}} j_{\mu}(\mathbf{x}) J^{\mu}(\mathbf{x})
$$

where $G=1.1664 \times 10^{-5} \mathrm{GeV}^{-2}$ is the Fermi weak coupling constant. $j_{\mu}$ and $J^{\mu}$ denote the leptonic and hadronic currents, respectively. According to V-A theory, the leptonic current takes the form

$$
j_{\mu}=\bar{\psi}_{v_{\ell}}(x) \gamma_{\mu}\left(1-\gamma_{5}\right) \psi_{v_{\ell}}(x),
$$

where $\psi_{v_{\ell}}$ are the neutrino/antineutrino spinors. 
From a nuclear physics point of view only the hadronic current is important. The structure for neutral-current processes of both vector and axial-vector components (neglecting the pseudo-scalar contributions) is written as

$$
J_{\mu}=\bar{\Psi}_{N}\left[F_{1} \gamma_{\mu}+F_{2} \frac{i \sigma_{\mu \nu} q^{\nu}}{2 M}+F_{A} \gamma_{\mu} \gamma_{5}\right] \Psi_{N}
$$

( $M$ stands for the nucleon mass and $\Psi_{N}$ denote the nucleon spinors). $F_{i}, i=1,2$, represent the weak nucleon form factors given in terms of the well-known charge and electromagnetic form factors (CVC theory) for proton $\left(F_{i}^{p}\right)$ and neutron $\left(F_{i}^{n}\right)$ by the expressions [44]

$$
F_{1,2}=\left(\frac{1}{2}-\sin ^{2} \theta_{W}\right)\left[F_{1,2}^{p}-F_{1,2}^{n}\right] \tau_{0}-\sin ^{2} \theta_{W}\left[F_{1,2}^{p}+F_{1,2}^{n}\right]
$$

Here $\tau_{0}$ represents the nucleon isospin operator and $\theta_{W}$ is the Weinberg angle $\left(\sin ^{2} \theta_{W}=\right.$ 0.2325). In (3.3) $F_{A}$ stands for the axial-vector form factor for which we employ the dipole ansatz given by

$$
F_{A}=-\frac{1}{2} g_{A}\left(\frac{1-q^{2}}{M_{A}^{2}}\right)^{-2} \tau_{0}
$$

where $M_{A}=1.05 \mathrm{GeV}$ is the dipole mass and $g_{A}=1.258$ is the static value (at $q=0$ ) of the axial form factor.

In the convention we used in the present work $q^{2}$, the square of the momentum transfer, is written as

$$
q^{2}=q^{\mu} q_{\mu}=\omega^{2}-\mathbf{q}^{2}=\left(\varepsilon_{i}-\varepsilon_{f}\right)^{2}-\left(\mathbf{p}_{i}-\mathbf{p}_{f}\right)^{2}
$$

where $\omega=\varepsilon_{i}-\varepsilon_{f}$ is the excitation energy of the nucleus. $\varepsilon_{i}$ denotes the energy of the incoming and $\varepsilon_{f}$ that of the outgoing neutrino. $\mathbf{p}_{i}, \mathbf{p}_{f}$ are the corresponding 3-momenta of the incoming and outgoing neutrino/antineutrino, respectively. In (3.4) we have not taken into account the strange quark contributions in the form factors. In the scattering reaction considered in this work only low-momentum transfers are involved and the contributions from strangeness can be neglected [45].

The neutral-current neutrino/antineutrino-nucleus differential cross section, after applying a multipole analysis of the weak hadronic current as in [46], is written as

$$
\left(\frac{\mathrm{d}^{2} \sigma_{i \rightarrow f}}{\mathrm{~d} \Omega \mathrm{d} \omega}\right)_{\nu / \bar{v}}=\frac{G^{2}}{\pi} \frac{\left|\vec{p}_{f}\right| \varepsilon_{f}}{\left(2 J_{i}+1\right)}\left(\sum_{J=0}^{\infty} \sigma_{C L}^{J}+\sum_{J=1}^{\infty} \sigma_{T}^{J}\right)
$$

The summations in (3.7) contain the contributions $\sigma_{C L}^{J}$, for the Coulomb $\widehat{\mathscr{M}}_{J}$ and longitudinal $\widehat{\iota}_{J}$, and $\sigma_{T}^{J}$, for the transverse electric $\hat{\tau}_{J}^{\mathrm{el}}$ and magnetic $\hat{\tau}_{J}^{\mathrm{mag}}$ multipole operators defined 
as in [47]. These operators include both polar-vector and axial-vector weak interaction components. The contributions $\sigma_{C L}^{J}$ and $\sigma_{T}^{J}$ are written as

$$
\begin{aligned}
\sigma_{C L}^{J}= & (1+\cos \theta)\left|\left\langle J_{f} \| \widehat{\mathcal{M}}_{J}(q)|| J_{i}\right\rangle\right|^{2}+\left(1+\cos \theta-2 b \sin ^{2} \theta\right)\left|\left\langle J_{f}\left\|\widehat{\mathcal{L}}_{J}(q)\right\| J_{i}\right\rangle\right|^{2} \\
& +\left[\frac{\omega}{q}(1+\cos \theta)\right] 2 \mathfrak{R} e\left\langle J_{f}\left\|\widehat{\mathcal{L}}_{J}(q)\right\| J_{i}\right\rangle\left\langle J_{f}\left\|\widehat{\mathcal{M}}_{J}(q)\right\| J_{i}\right\rangle^{*}, \\
\sigma_{T}^{J}= & \left(1-\cos \theta+b \sin ^{2} \theta\right)\left[\left|\left\langle J_{f}\left\|\hat{\mathcal{\tau}}_{J}^{\mathrm{mag}}(q)\right\| J_{i}\right\rangle\right|^{2}+\left|\left\langle J_{f}\left\|\hat{\mathcal{\tau}}_{J}^{\mathrm{el}}(q)\right\| J_{i}\right\rangle\right|^{2}\right] \\
\mp & \frac{\left(\varepsilon_{i}+\varepsilon_{f}\right)}{q}(1-\cos \theta) 2 \Re e\left\langle J_{f}\left\|\hat{\mathcal{\tau}}_{J}^{\mathrm{mag}}(q)\right\| J_{i}\right\rangle\left\langle J_{f}\left\|\hat{\mathcal{\tau}}_{J}^{\mathrm{el}}(q)\right\| J_{i}\right\rangle^{*},
\end{aligned}
$$

where $\theta$ denotes the outgoing neutrino scattering angle and $b=\varepsilon_{i} \varepsilon_{f} / q^{2}$. In (3.9) the - sign corresponds to neutrino scattering and the + sign to antineutrino one.

\section{Energies and Wave Functions}

For neutral current neutrino-nucleus-induced reactions, the ground state and the excited states of the even-even nucleus are created using the quasi-particle random phase approximation (QRPA) including two quasi-neutron and two quasi-proton excitations in the QRPA matrix [48] (hereafter denoted by pp-nn QRPA). We start by writing the A-fermion Hamiltonian $\mathrm{H}$, in the occupation number representation, as a sum of two terms. One is the sum of the single-particle energies (spe) $\epsilon_{\alpha}$ which runs over all values of quantum numbers $\alpha \equiv\left\{n_{\alpha}, l_{\alpha}, j_{\alpha}, m_{\alpha}\right\}$ and the second term which includes the two-body interaction $V$, that is

$$
H=\sum_{\alpha} \epsilon_{\alpha} c_{\alpha}^{\dagger} c_{\alpha}+\frac{1}{4} \sum_{\alpha \beta \gamma \delta} \bar{V}_{\alpha \beta \gamma \delta} c_{\alpha}^{\dagger} c_{\beta}^{\dagger} c_{\delta} c_{\gamma}
$$

where the two-body term contains the antisymmetrised two-body interaction matrix element defined by $\bar{V}_{\alpha \beta \gamma \delta}=\langle\alpha \beta|V| \gamma \delta\rangle-\langle\alpha \beta|V| \delta \gamma\rangle$. The operators $c_{\alpha}^{\dagger}$ and $c_{\alpha}$ stand for the usual creation and destruction operators of nucleons in the state $\alpha$.

For spherical nuclei with partially filled shells, the most important effect of the twobody force is to produce pairing correlations. The pairing interaction is taken into account by using the BCS theory [49]. The simplest way to introduce these correlations in the wave function is to perform the Bogoliubov-Valatin transformation

$$
\begin{aligned}
& a_{\alpha}^{\dagger}=u_{\alpha} c_{\alpha}^{\dagger}-v_{\alpha} \tilde{c}_{\alpha} \\
& \tilde{a}_{\alpha}^{\dagger}=u_{\alpha} \widetilde{c}_{\alpha}^{\dagger}+v_{\alpha} c_{\alpha}
\end{aligned}
$$

where $\tilde{c}_{\alpha}^{\dagger}=c_{-\alpha}^{\dagger}(-1)^{j_{\alpha}+m_{\alpha}}, \tilde{a}_{\alpha}^{\dagger}=a_{-\alpha}^{\dagger}(-1)^{j_{\alpha}+m_{\alpha}}$ and $-\alpha \equiv\left\{n_{\alpha}, l_{\alpha}, j_{\alpha},-m_{\alpha}\right\}$. The occupation amplitudes $v_{\alpha}$ and $u_{\alpha}$ are determined via variational procedure for minimizing the energy of the BCS ground state for protons and neutrons separately. In the BCS approach the ground 
state of an even-even nucleus is described as a superconducting medium where all the nucleons have formed pairs that effectively act as bosons. The BCS ground state is defined as

$$
|\mathrm{BCS}\rangle=\prod_{\alpha>0}\left(u_{\alpha}-v_{\alpha} c_{\alpha}^{\dagger} \widetilde{c}_{\alpha}^{\dagger}\right)|\mathrm{CORE}\rangle,
$$

where $|C O R E\rangle$ represents the nuclear core (effective particle vacuum).

After the transformation (4.2) the Hamiltonian can be written in its quasi-particle representation as

$$
H=\sum_{\alpha} E_{\alpha} a_{\alpha}^{\dagger} a_{\alpha}+H_{\mathrm{qp}}
$$

where the first term gives the single quasi-particle energies $E_{\alpha}$ and the second one includes the different components of the residual interaction.

In the present calculations we use a renormalization parameter $g_{\text {pair }}$ which can be adjusted when doing the BCS calculations. The monopole matrix elements $\langle\alpha \alpha ; J=$ $0|V| \beta \beta ; J=0\rangle$ of the two-body interaction are multiplied by a factor $g_{\text {pair }}$. The adjustment can be done by comparing the resulting lowest quasi-particle energy to the phenomenological energy gap $\Delta$ obtained from the separation energies of the neighboring doubly-even nuclei for protons and neutrons separately.

In the next step the excited states of the even-even reference nucleus are constructed by use of the QRPA. In the QRPA the creation operator for an excited state (QRPA phonon) has the form

$$
\widehat{Q}^{\dagger}\left(J_{k}^{\pi} M\right)=\sum_{\alpha \leq \alpha^{\prime}}\left[X_{\alpha \alpha^{\prime}}^{J_{k}^{\pi}} A^{\dagger}\left(\alpha \alpha^{\prime} ; J M\right)-Y_{\alpha \alpha^{\prime}}^{J_{k}^{\pi}} \widetilde{A}\left(\alpha \alpha^{\prime} ; J M\right)\right]
$$

where the quasi-particle pair creation $A^{\dagger}\left(\alpha \alpha^{\prime} ; J M\right)$ and annihilation $\tilde{A}\left(\alpha \alpha^{\prime} ; J M\right)$ operators are defined as

$$
\begin{aligned}
& A^{\dagger}\left(\alpha \alpha^{\prime} ; J M\right) \equiv\left(1+\delta_{\alpha \alpha^{\prime}}\right)^{-1 / 2}\left[a_{\alpha}^{\dagger} a_{\alpha^{\prime}}^{\dagger}\right]_{J M^{\prime}} \\
& \tilde{A}\left(\alpha \alpha^{\prime} ; J M\right) \equiv(-1)^{J+M} A\left(\alpha \alpha^{\prime} ; J-M\right),
\end{aligned}
$$

where $\alpha$ and $\alpha^{\prime}$ are either proton $(p)$ or neutron $(n)$ indices, $M$ labels the magnetic substates, and $k$ numbers the states for particular angular momentum $J$ and parity $\pi$.

The $X$ and $Y$ forward and backward going amplitudes are determined from the QRPA matrix equation

$$
\left(\begin{array}{cc}
\mathcal{A} & B \\
-B & -\mathcal{A}
\end{array}\right)\left(\begin{array}{l}
X^{J^{\pi}} \\
Y^{J^{\pi}}
\end{array}\right)=\omega\left(\begin{array}{l}
X^{J^{\pi}} \\
Y^{J^{\pi}}
\end{array}\right)
$$


where $\omega$ denotes the excitation energies of the nuclear state $\left|J^{\pi}\right\rangle$. The QRPA matrices $\mathcal{A}$ and $B$, are deduced by the matrix elements of the double commutators of $A^{\dagger}$ and $A$ with the nuclear hamiltonian $\widehat{H}$ defined as

$$
\begin{aligned}
& \mathcal{A}_{J}\left(\alpha \alpha^{\prime} ; \beta \beta^{\prime}\right)=\left\langle\mathrm{BCS}\left|\left[A\left(\alpha \alpha^{\prime} ; J M\right), \widehat{H}, A^{\dagger}\left(\beta \beta^{\prime} ; J M\right)\right]\right| \mathrm{BCS}\right\rangle, \\
& \mathcal{B}_{J}\left(\alpha \alpha^{\prime} ; \beta \beta^{\prime}\right)=-\left\langle\mathrm{BCS}\left|\left[A\left(\alpha \alpha^{\prime} ; J M\right), \widehat{H}, \tilde{A}\left(\beta \beta^{\prime} ; J M\right)\right]\right| \mathrm{BCS}\right\rangle,
\end{aligned}
$$

where $2[A, B, C]=[A,[B, C]]+[[A, B], C]$. Finally the two-body matrix elements of each multipolarity $J^{\pi}$, occurring in the QRPA matrices $\mathcal{A}$ and $B$, are multiplied by two phenomenological scaling constants, namely, the particle-hole strength $g_{\mathrm{ph}}$ and the particleparticle strength $g_{\text {pp }}$. These parameter values are determined by comparing the resulting lowest phonon energy with the corresponding lowest collective vibrational excitation of the doubly-even nucleus and by reproducing some giant resonances which play crucial role.

\section{Results}

\subsection{Calculated Cross Sections}

In order to investigate neutrino scattering off the ${ }^{40} \mathrm{Ar}$ and ${ }^{132} \mathrm{Xe}$ nuclei we followed the procedure of [30-34]. Specifically we have performed explicit state-by-state calculations for the nuclear transition matrix elements given by (3.8) and (3.9) in the framework of QRPA. The initial nucleus was assumed to be spherically symmetric having a $0^{+}$ground state. In the case of ${ }^{132} \mathrm{Xe}$ we have adopted ${ }^{40} \mathrm{Ca}$ as inert core and the two oscillator $3 \hbar \omega$ and $4 \hbar \omega$ major shells, plus the intruder orbital $h_{11 / 2}$ from the next higher oscillator major shell, as valence space for protons and neutrons. In the case of ${ }^{40} \mathrm{Ar}$ we have considered the major shells $0,1,2$, and $3 \hbar \omega$ as the model space for both protons and neutrons. The corresponding single-particle energies (s.p.e) were produced by the Coulomb corrected Woods-Saxon potential using the parameters of Bohr and Mottelson [50].

The two-body interaction matrix elements were obtained from the Bonn one-bosonexchange potential applying G-matrix techniques [51]. The strong pairing interaction between the nucleons can be adjusted by solving the BCS equations. The monopole matrix elements of the two-body interaction are scaled by the pairing strength parameters $g_{\text {pair }}^{p}$ and $g_{\text {pair }}^{n}$ separately for protons and neutrons. The adjustment can be done by comparing the resulting lowest quasiparticle energy to reproduce the phenomenological pairing gap obtained by using the linear approximation [52]

$$
\begin{aligned}
& \Delta_{n}\left({ }_{Z}^{A} X\right)=-\frac{1}{4}\left[S_{n}\left({ }_{Z}^{A+1} X\right)-2 S_{n}\left({ }_{Z}^{A} X\right)+S_{n}\left({ }_{Z}^{A-1} X\right)\right], \\
& \Delta_{p}\left({ }_{Z}^{A} X\right)=-\frac{1}{4}\left[S_{p}\left({ }_{Z+1}^{A+1} X\right)-2 S_{p}\left({ }_{Z}^{A} X\right)+S_{p}\left({ }_{Z-1}^{A-1} X\right)\right]
\end{aligned}
$$

in which ${ }_{Z}^{A} X$ stands for the doubly-even nucleus under consideration. The separation energies $S_{n / p}$ are provided by [53]. The results of this procedure lead to the pairing-strength parameters $g_{\text {pair }}^{p}=1.05$ and $g_{\text {pair }}^{n}=0.88$ for ${ }^{40} \mathrm{Ar}$ and $g_{\text {pair }}^{p}=1.08$ and $g_{\text {pair }}^{n}=0.89$ for ${ }^{132}$ Xe. After settling the values of the pairing parameters, two other parameters are left to fix, the 
overall scale of the particle-hole interaction $g_{\text {ph }}$ and separately the particle-particle channel of the interaction $g_{\mathrm{pp}}$ for each multipole up to $J=8^{ \pm}$. The QRPA parameters are determined so that the low-lying energy spectrum fits to the experimental data [30-34]. An alternative fixing of the parameters $g_{\mathrm{ph}}$ and $g_{\mathrm{pp}}$, especially for the charged-current neutrino-nucleus reactions, could be done on the giant dipole resonance of the studied nucleus. Using the formalism for the double differential cross section we have calculated $\mathrm{d}^{2} \sigma_{i \rightarrow f} / \mathrm{d} \Omega \mathrm{d} \omega$ (see (3.7)) for all QRPA states up to $40 \mathrm{MeV}$, in contrast to previous RPA calculations, which consider only a few states known by experiment [42]. The total cross section $\sigma\left(E_{v}\right)$ was obtained by integrating over the scattering angles $\theta$ and $\phi$ and subsequently summing over all discrete final sates. The results were obtained for coherent $0_{\mathrm{gs}}^{+} \rightarrow 0_{\mathrm{gs}}^{+}$cross sections (elastic channel) as well as for incoherent cross sections $0_{\mathrm{gs}}^{+} \rightarrow J_{f}$ (inelastic channel).

The coherent neutrino-nucleus scattering (CNNS) is an important prediction of the Standard Model. It is worth mentioning that there is quite a wide literature describing CNNS mainly based on nuclear recoil signals [54]. The differential cross section versus neutrino energy $E_{v}$ is given by [55]

$$
\left(\frac{d \sigma}{d \Omega}\right)_{\mathrm{coh}}\left(E_{v}\right)=\frac{G^{2}}{16 \pi^{2}} E_{v}^{2}(1+\cos \theta) Q_{W}^{2} F^{2}\left(q^{2}\right), \quad q^{2}=2 E_{v}^{2}(1-\cos \theta)
$$

where $\theta$ denotes the scattering angle of the incident neutrino in the lab frame of the recoil nucleus, $G$ is the Fermi constant, and $Q_{W}$ is the weak charge of the nucleus with $N$ neutrons and $Z$ protons:

$$
Q_{W}=N-\left(1-4 \sin ^{2} \Theta_{W}\right) Z
$$

with $\Theta_{W}$ being the weak mixing angle $\left(\sin ^{2} \Theta_{W} \approx 0.231\right) . F\left(q^{2}\right)$ stands for the elastic form factor [56] that describes the distribution of weak charge within the nucleus. Integrating the differential cross section with respect to $d \Omega$ we obtain the CNNS cross section as a function of the neutrino energy $E_{v}$

$$
\sigma_{\mathrm{coh}}\left(E_{v}\right)=\int\left(\frac{d \sigma}{d \Omega}\right)_{\mathrm{coh}}\left(E_{v}\right) d \Omega
$$

Figure 4 shows the contributions of coherent and incoherent cross sections as a function of the incoming neutrino energies taken from the QRPA calculations. In Figures 4(a) and $4(\mathrm{~b})$ we also present the total cross sections (coherent plus incoherent) for the reactions ${ }^{132} \mathrm{Xe}\left(v, v^{\prime}\right){ }^{132} \mathrm{Xe}$ and ${ }^{40} \mathrm{Ar}\left(v, v^{\prime}\right){ }^{40} \mathrm{Ar}$, respectively. As it is seen, the coherent cross sections are greater than incoherent ones by at least an order of magnitude in the relevant energy region and dominates the total cross section for all neutrino energies $E_{v} \leq 100 \mathrm{MeV}$. These results are similar to the calculations performed by other nuclear systems [57]. In Figure 4 we also present the results for the coherent channel taken from (5.4). As it is seen, the coherent $0_{\mathrm{gS}}^{+} \rightarrow 0_{\mathrm{gS}}^{+}$cross sections obtained from QRPA are in agreement with those taken from (5.4), especially for neutrinos with energies below $40 \mathrm{MeV}$. The theoretical uncertainty on the neutrino-nucleus scattering cross section comes from nuclear modelling in the form factor calculation. 


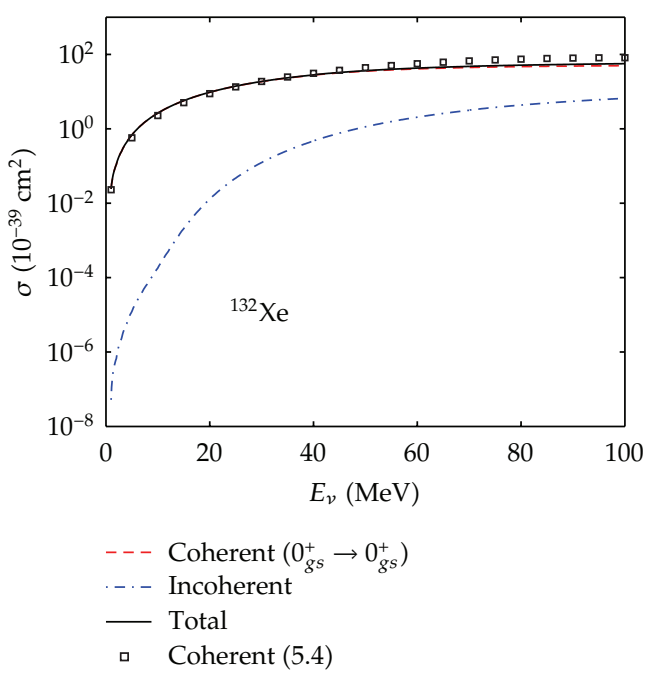

(a)

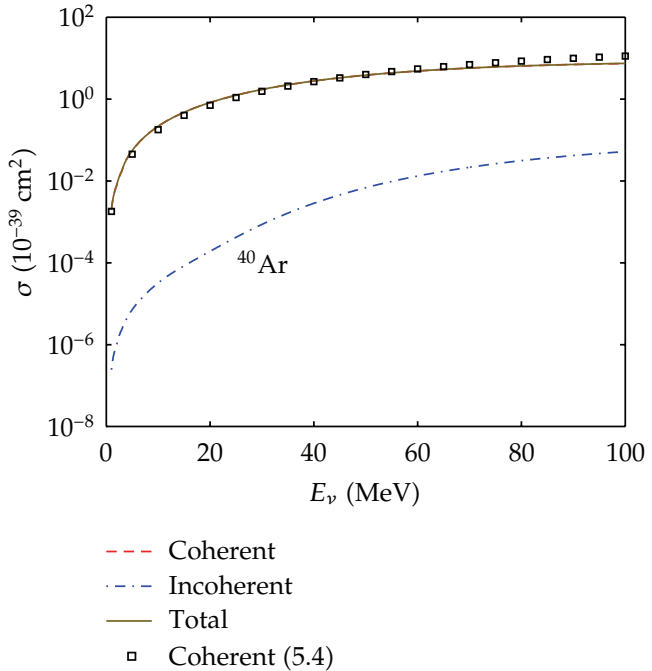

(b)

Figure 4: (Color on line). Coherent, incoherent, and total cross section as a function of the incoming neutrino energy $E_{v}$, in the NC reactions ${ }^{132} \mathrm{Xe}\left(v, v^{\prime}\right){ }^{132} \mathrm{Xe}(\mathrm{a})$ and ${ }^{40} \operatorname{Ar}\left(v, v^{\prime}\right){ }^{40} \mathrm{Ar}(\mathrm{b})$.

Figure 5 illustrates the corresponding distribution of the different multipolarities to the incoherent cross section for two impinging neutrino energies. As it is seen, in low-energy region, the transitions $0^{+}$for ${ }^{40} \mathrm{Ar}$ and $1^{-}$for ${ }^{132} \mathrm{Xe}$ are the most pronounced channels. On the other hand, in high-energy region, the incoherent scattering for ${ }^{40} \mathrm{Ar}$ is dominated mostly by the $1^{-}$transition while other transitions like $3^{-}$and $2^{+}$start to contribute significantly. In the case of ${ }^{132} \mathrm{Xe}$ the channels $1^{-}, 3^{-}, 2^{+}$, and $4^{+}$are dominant.

In order to obtain more information about supernova neutrinos, the total cross section $\sigma\left(E_{v}\right)$ has to be folded with the FD neutrino energy distribution. The individual contributions into coherent, incoherent, and total (coherent plus incoherent) cross sections are given in Table 4 . As it is seen from this table the coherent scattering clearly dominates the total cross sections. Finally in Table 5 we compare our results for the coherent $0_{\mathrm{gs}}^{+} \rightarrow 0_{\mathrm{gS}}^{+}$cross sections folded with the FD spectra with those obtained from (5.4). As it is seen, the results obtained by means of the standard formula (5.4) are consistent with those taken by QRPA calculations. It is clear that the main contribution to the coherent channel comes from the transition $0_{\mathrm{gs}}^{+} \rightarrow$ $0_{\mathrm{gs}}^{+}$.

\subsection{Neutrino Detection with a TPC Detector}

One of the most famous detectors for dedicated supernova detection is gaseous spherical TPC detector (Time Projection Chamber) [58]. TPC detector allows measurements of high multiplicity events $(\simeq 200)$ coming from relativistic nucleus-nucleus collisions. It has low threshold and high resolution. As it is known, a spherical TPC detector filled with either Xe or Ar has been proposed as a device able to detect low-energy neutrinos as those coming from a galactic supernova and, in particular, it will be able to observe coherent neutrino-nucleus scattering [59-64].

Taking into account our results concerning the total cross sections for Ar and Xe, it is a good opportunity to employ and test the spherical TPC gaseous detector of volume $V$ 


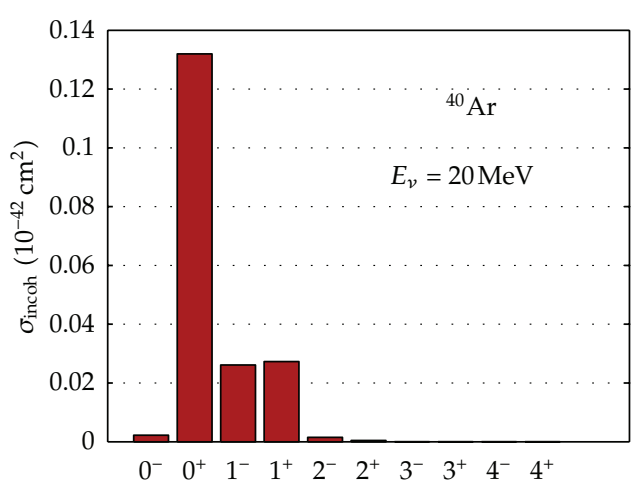

(a)

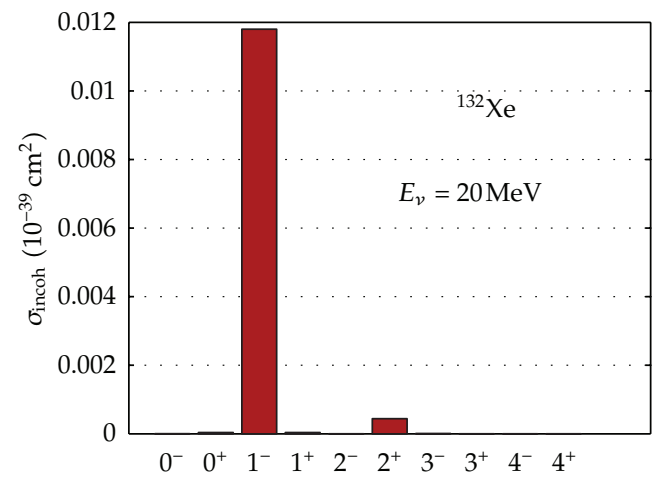

(c)

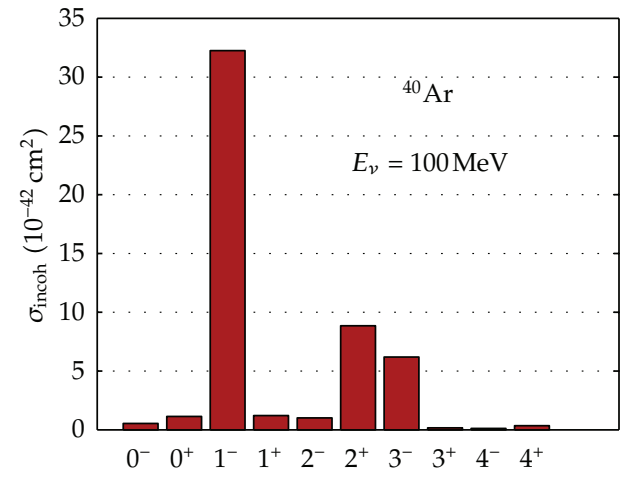

(b)

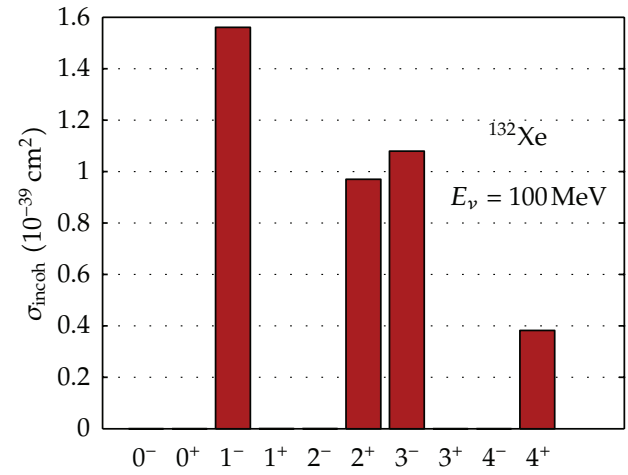

(d)

Figure 5: (Color on line). Partial multipole distributions to the incoherent cross sections for ${ }^{40} \mathrm{Ar}$ and ${ }^{132} \mathrm{Xe}$, at the incoming neutrino energies $E_{v}=20$ and $100 \mathrm{MeV}$.

under pressure $P$ and temperature $T_{0}$, filled with noble gas such as $\mathrm{Ar}$ and $\mathrm{Xe}$. In this case, the number of expected events in a year takes the form

$$
\mathcal{R}=3.156 \times 10^{7} \frac{t}{1 y} \Phi(v, L=50 \mathrm{~m}) \sigma_{\text {tot }} \frac{P V}{k T_{0}} s(V, L),
$$

where the parameter $s(V, L)$ is a geometrical factor needed when a large detector is close to the source [65]. It depends on the shape of the vessel and the distance $L$ of its geometric center from the source. In the case of sphere of radius $R$ with its center at a distance $L$ from the source, the function $s(V, R)$ depends only on the ratio $R / L$ and it is given by

$$
\begin{gathered}
s(R / L)=\frac{L^{2}}{(4 / 3) \pi R^{3}} 2 \pi L \int_{0}^{R / L} x^{2} d x \\
\int_{0}^{\pi} d \theta \frac{\sin \theta}{1+x^{2}+2 x \cos \theta}, \quad x=\frac{r}{L} .
\end{gathered}
$$

Spherical coordinates $(r, \theta, \phi)$ are used to specify any point inside the sphere. The origin of coordinates was chosen at the center of the sphere with polar axis being the straight line from 
Table 4: Coherent, incoherent, and total (coherent plus incoherent) neutrino nucleus cross section for ${ }^{40} \mathrm{Ar}$ and ${ }^{132} \mathrm{Xe}$ targets. The supernova neutrino spectra described by a two-parameter Fermi-Dirac distribution with $\alpha=0$ and 3 for various temperatures $T(\mathrm{MeV})$.

\begin{tabular}{lllllll}
\hline$(T, \alpha)$ & $(3.5,0)$ & $(5,0)$ & $(8,0)$ & $(2.75,3)$ & $(4,3)$ & $(6,3)$ \\
\hline \multicolumn{7}{c}{$\sigma_{\text {coh }}\left(\right.$ in $\left.10^{-39} \mathrm{~cm}^{2}\right)$} \\
\hline${ }^{40} \mathrm{Ar}$ & 0.33 & 0.63 & 1.40 & 0.30 & 0.61 & 1.26 \\
${ }^{132} \mathrm{Xe}$ & 3.87 & 7.17 & 14.54 & 3.63 & 7.09 & 13.53 \\
\hline \multicolumn{7}{c}{$\sigma_{\text {incoh }}\left(\right.$ in $\left.10^{-42} \mathrm{~cm}^{2}\right)$} \\
\hline${ }^{40} \mathrm{Ar}$ & 0.08 & 0.28 & 1.56 & 0.06 & 0.21 & 0.95 \\
${ }^{132} \mathrm{Xe}$ & 6.37 & 36.23 & 239.40 & 3.19 & 23.10 & 143.10 \\
\hline \multicolumn{7}{c}{$\sigma_{v}=\sigma_{\text {coh }}+\sigma_{\text {incoh }}\left(\right.$ in $\left.10^{-39} \mathrm{~cm}^{2}\right)$} \\
${ }^{40} \mathrm{Ar}$ & 0.32 & 0.63 & 1.40 & 0.30 & 0.61 & 1.26 \\
${ }^{132} \mathrm{Xe}$ & 3.87 & 7.20 & 14.78 & 3.64 & 7.11 & 13.68 \\
\hline
\end{tabular}

Table 5: Coherent neutrino nucleus cross sections calculated by (5.4) and by the QRPA approach (numbers in parenthesis) for ${ }^{40} \mathrm{Ar}$ and ${ }^{132} \mathrm{Xe}$ targets. The supernova neutrino spectra described by a two parameter Fermi-Dirac distribution with $\alpha=0$ and 3 for various temperatures $T(\mathrm{MeV})$.

\begin{tabular}{lcccccc}
\hline \multicolumn{7}{c}{$\sigma_{\mathrm{coh}}\left(\right.$ in $\left.10^{-39} \mathrm{~cm}^{2}\right)$} \\
\hline$(T, \alpha)$ & $(3.5,0)$ & $(5,0)$ & $(8,0)$ & $(2.75,3)$ & $(4,3)$ & $(6,3)$ \\
\hline${ }^{40} \mathrm{Ar}$ & $0.28(0.33)$ & $0.56(0.63)$ & $1.35(1.40)$ & $0.26(0.30)$ & $0.53(0.61)$ & $1.16(1.26)$ \\
${ }^{132} \mathrm{Xe}$ & $3.50(3.87)$ & $6.86(7.17)$ & $15.63(14.54)$ & $3.23(3.63)$ & $6.63(7.09)$ & $13.87(13.53)$ \\
\hline
\end{tabular}

the source to the center. With the above choice the flux is independent of the angle $\phi$. A plot of the function $s(R / L)$ is presented in Figure 6. The geometric factor $s(R / L)$ is close to unity in the actual experimental setup where $L \gg R$.

For a typical distance $L=50 \mathrm{~m}$ we can take as neutrino flux for each neutrino flavor the value $\Phi(v, L=50 \mathrm{~m})=1.95 \times 10^{6} \mathrm{~cm}^{-2} \mathrm{~s}^{-1}$. Summing over all the neutrino flavors we find the total cross sections $\sigma_{\text {tot }}=6.5 \times 10^{-39} \mathrm{~cm}^{2}$ for $A=40$ and $\sigma_{\text {tot }}=7.0 \times 10^{-38} \mathrm{~cm}^{2}$ for $A=132$. Finally the total number of events $\mathcal{R}$ in a year is calculated using (5.5) and listed in Table 6. The parameters considered in our calculations are consistent with the experimental works of [59-66]. Moreover, for a primary supernova neutrino flux (time averaged)

$$
\Phi_{v}=\frac{N_{v}}{4 \pi L^{2}}
$$

at a distance $L=10 \mathrm{Kpc}=3.1 \times 10^{22} \mathrm{~cm}$, the number of the observed events for each neutrino flavor is found to be

$$
N_{e v}=\Phi_{\nu} \sigma_{v} \frac{P V}{k T_{0}}
$$

In Table 7 the numbers of event rates are listed for two given radii $R=6$ and $9 \mathrm{~m}$. As it seen, employing ${ }^{132} \mathrm{Xe}$ as a target nucleus one expects about 1761 events for a sphere of radius $6 \mathrm{~m}$, while for ${ }^{40} \mathrm{Ar}$ one expects about 562 events but with a vessel of larger radius $(R=9 \mathrm{~m})$. 


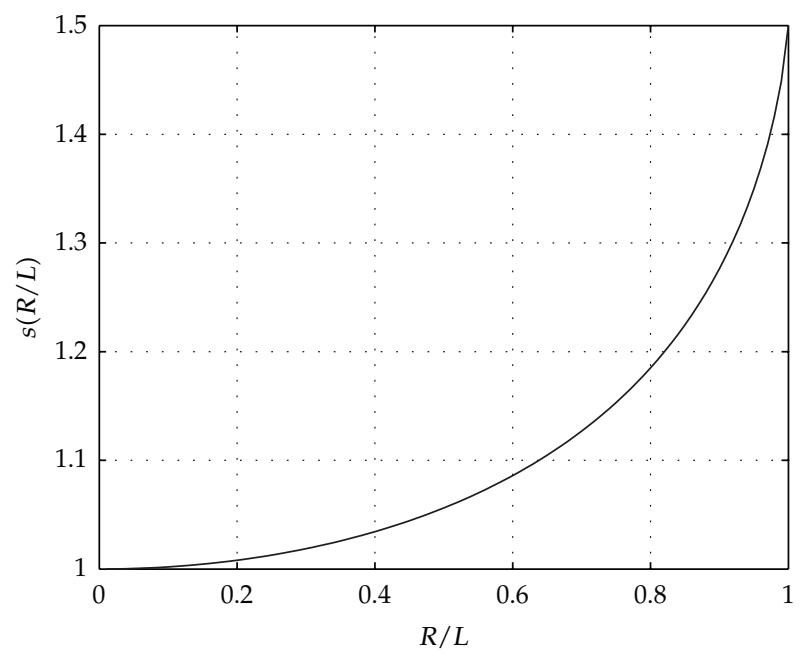

Figure 6: Geometrical factor $s(R / L)$ for a sphere of radius $R$ whose center is at distance $L$ from the source.

Table 6: Number of events in a year for a spherical detector of various radii $R$ with its center at a distance $L=50 \mathrm{~m}$ from the source. The vessel is filled with gas under pressure $P=10 \mathrm{Atm}$ and temperature $T_{0}=300 \mathrm{~K}$. The total cross sections are obtained summing over all neutrino flavors. The chemical potential of the neutrino spectra is taken as $\alpha=0$.

\begin{tabular}{lccccc}
\hline Target & $R=1 \mathrm{~m}$ & $R=3 \mathrm{~m}$ & $R=6 \mathrm{~m}$ & $R=9 \mathrm{~m}$ & $R=12 \mathrm{~m}$ \\
\hline${ }^{132} \mathrm{Xe}$ & $4.4 \times 10^{3}$ & $1.2 \times 10^{5}$ & $9.5 \times 10^{5}$ & $3.2 \times 10^{6}$ & $7.6 \times 10^{6}$ \\
${ }^{40} \mathrm{Ar}$ & $4.1 \times 10^{2}$ & $1.1 \times 10^{4}$ & $8.8 \times 10^{4}$ & $3.0 \times 10^{5}$ & $7.0 \times 10^{5}$ \\
\hline
\end{tabular}

Table 7: The number of events rate for a spherical detector of various radii $R$. The neutrinos are emitted from a supernova at a distance $L=10 \mathrm{Kpc}$. The spherical vessel of volume $V$ is filled with gas under pressure $P=10 \mathrm{Atm}$ and temperature $T_{0}=300 \mathrm{~K}$. The chemical potential of the neutrino spectra is taken as $\alpha=0$.

\begin{tabular}{|c|c|c|c|c|c|}
\hline \multicolumn{6}{|c|}{$N_{e v}$} \\
\hline & $R(\mathrm{~m})$ & $v_{e}$ & $\bar{v}_{e}$ & $\sum_{x} v_{x}$ & Total \\
\hline \multirow{2}{*}{${ }^{40} \mathrm{Ar}$} & 6 & 16 & 22 & 127 & 165 \\
\hline & 9 & 56 & 76 & 430 & 562 \\
\hline \multirow{2}{*}{${ }^{132} \mathrm{Xe}$} & 6 & 197 & 255 & 1309 & 1761 \\
\hline & 9 & 666 & 861 & 4418 & 5945 \\
\hline
\end{tabular}

\section{Conclusions}

In this paper the coherent and incoherent contribution in neutrino-nucleus scattering due to neutral current has been examined considering as target materials the isotopes ${ }^{40} \mathrm{Ar}$ and ${ }^{132} \mathrm{Xe}$. The differential as well as the total cross sections have been derived employing the quasi-particle random phase approximation. In order to obtain information appropriate for describing terrestrial detection of supernova neutrinos, the total cross sections (coherent+incoherent) were folded with a neutrino energy spectrum in the FD model. An enhancement of the neutral current component is achieved via the coherent channel $\left(0_{\mathrm{gs}}^{+} \rightarrow\right.$ $0_{\mathrm{gs}}^{+}$) which is dominant with respect to incoherent one. 
From the above results one can test a gaseous spherical TPC detector dedicated for $\mathrm{SN}$ neutrino detection. Filling the TPC detector with the noble gas ${ }^{132} \mathrm{Xe}$ under pressure $P=$ $10 \mathrm{Atm}$ and temperature $T_{0}=300 \mathrm{~K}$ one expects about 1761 events for a sphere of radius $6 \mathrm{~m}$. Employing ${ }^{40} \mathrm{Ar}$ one expects 562 events but with a vessel of larger radius $(R=9 \mathrm{~m})$. This detector can also be tested with earth neutrino sources, which have a neutrino spectrum analogous to that of an SN. Neutral current detectors, which are not sensitive to neutrino oscillation effects, could provide a great deal of information about the primary supernova neutrino flux.

\section{Acknowledgment}

The author would like to thank Professor J. D. Vergados for useful discussions.

\section{References}

[1] T. W. Donnelly and R. D. Peccei, “Neutral current effects in nuclei,” Physics Reports, vol. 50, no. 1, pp. 1-85, 1979.

[2] R. Davis, "A review of the Homestake solar neutrino experiment," Progress in Particle and Nuclear Physics, vol. 32, pp. 13-32, 1994.

[3] K. Langanke, "Weak interaction, nuclear physics and supernovae," Acta Physica Polonica B, vol. 39, no. 2, pp. 265-281, 2008.

[4] W. C. Haxton, "Radiochemical neutrino detection via ${ }^{127} \mathrm{I}\left(v_{e}, e^{-}\right){ }^{127} \mathrm{Xe}$, ” Physical Review Letters, vol. 60, no. 9, pp. 768-771, 1988.

[5] J. N. Bahcall and R. K. Ulrich, "Solar models, neutrino experiments, and helioseismology," Reviews of Modern Physics, vol. 60, no. 2, pp. 297-372, 1988.

[6] K. Kubodera and S. Nozawa, "Neutrino-nucleus reactions," International Journal of Modern Physics E, vol. 3, no. 1, pp. 101-148, 1994.

[7] J. Rapaport, T. Taddeucci, P. Welch et al., "Empirical evaluation of Gamow-Teller strength function for ${ }^{37} \mathrm{CI} \rightarrow{ }^{37} \mathrm{AR}$ and its implication in the cross section for solar neutrino absorption by ${ }^{37} \mathrm{Cl}, "$ Physical Review Letters, vol. 47, no. 21, pp. 1518-1521, 1981.

[8] J. Rapaport, P. Welch, J. Bahcall et al., "Solar-Neutrino detection: experimental determination of Gamow-Teller strengths via the ${ }^{98} \mathrm{Mo}$ and ${ }^{115} \mathrm{In}(p, n)$ reactions," Physical Review Letters, vol. 54 , no. 21, pp. 2325-2328, 1985.

[9] D. Krofcheck, E. Sugarbaker, J. Rapaport et al., “Gamow-Teller strength function in ${ }^{71}$ Ge via the $(p, n)$ reaction at medium energies," Physical Review Letters, vol. 55, no. 10, pp. 1051-1054, 1985.

[10] D. Krofcheck, E. Sugarbaker, A. J. Wagner et al., "Gamow-Teller strength distribution in ${ }^{81} \mathrm{Kr}$ and the consequences for a ${ }^{81} \mathrm{Br}$ solar neutrino detector," Physics Letters B, vol. 189, no. 3, pp. 299-303, 1987.

[11] Y. S. Lutostansky and N. B. Shulgina, "Strength function of ${ }^{127}$ Xe and iodine-xenon neutrino detector," Physical Review Letters, vol. 67, no. 4, pp. 430-432, 1991.

[12] J. W. F. Valle, “Neutrinos in astroparticle physics," AIP Conference Proceedings, vol. 878, pp. 369-384, 2006.

[13] B. Bodmann, N. E. Booth, F. Burtak et al., "First observation of the neutral current nuclear excitation ${ }^{12} \mathrm{C}\left(\mathrm{v}, \mathrm{v}^{\prime}\right){ }^{12} \mathrm{c}^{*}\left(1^{+}, 1\right), "$ Physics Letters, Section B, vol. 267, no. 3, pp. 321-324, 1991.

[14] B. Bodmann, N. E. Booth, F. Burtak et al., "Cross section of the charged current reaction ${ }^{12} \mathrm{C}\left(v_{e}, e^{-}\right){ }^{12}$ Ng.s.," Physics Letters, Section B, vol. 280, no. 3-4, pp. 198-203, 1992.

[15] B. E. Bodmann, N. B. Booth, G. Drexlin et al., "Neutrino interactions with carbon: recent measurements and a new test of electron-neutrino, anti-muon-neutrino universalit," Physics Letters, Section B, vol. 332, no. 3-4, pp. 251-257, 1994.

[16] G. Drexlin, "KARMEN: precision tests of the standard model with neutrinos from muon and pion decay," Progress in Particle and Nuclear Physics, vol. 32, pp. 375-396, 1994.

[17] C. Volpe, N. Auerbach, and N. Van Giai, "Charged-current neutrino- ${ }^{208} \mathrm{~Pb}$ reactions," Physical Review C, vol. 65, no. 4, Article ID 044603, 5 pages, 2002.

[18] T. W. Donnelly and J. D. Walecka, "Semi-leptonic weak and electromagnetic interactions in nuclei with application to ${ }^{16} \mathrm{O}$," Physics Letters B, vol. 41, no. 3, pp. 275-280, 1972. 
[19] T. W. Donnelly, "Low-energy neutrino reactions with ${ }^{12} \mathrm{C}$ and ${ }^{16} \mathrm{O}$, , Physics Letters B, vol. 43, no. 2, pp. 93-97, 1973.

[20] J. B. Langworthy, B. A. Lamers, and H. Überall, "Inelastic neutrino reactions on ${ }^{16} \mathrm{O}$," Nuclear Physics, Section A, vol. 280, no. 2, pp. 351-364, 1977.

[21] E. V. Bugaev, G. S. Bisnovatyi-Kogan, M. A. Rudzsky, and Z. F. Seidov, "The interaction of intermediate energy neutrinos with nuclei," Nuclear Physics, Section A, vol. 324, no. 2-3, pp. 350-364, 1979.

[22] J. S. Bell and C. H. Llewellyn-Smith, “Quasielastic neutrino-nucleus interactions,” Nuclear Physics B, vol. 28, no. 2, pp. 317-340, 1971.

[23] T. K. Gaisser and J. S. O'Connell, "Interactions of atmospheric neutrinos in nuclei at low energy," Physical Review D, vol. 34, no. 3, pp. 822-825, 1986.

[24] T. Kuramoto, M. Fukugita, Y. Kohyama, and K. Kubodera, “Neutrino-induced reaction cross sections at intermediate energies for chlorine and water detectors," Nuclear Physics, Section A, vol. 512, no. 4, pp. 711-736, 1990.

[25] S. K. Singh and E. Oset, "Quasielastic neutrino (antineutrino) reactions in nuclei and the axial-vector form factor of the nucleon," Nuclear Physics, Section A, vol. 542, no. 4, pp. 587-615, 1992.

[26] E. Kolbe, "Differential cross sections for neutrino scattering on ${ }^{12} \mathrm{C}$," Physical Review C, vol. 54, no. 4, pp. 1741-1748, 1996.

[27] P. C. Divari, T. S. Kosmas, J. D. Vergados, and L. D. Skouras, "Shell model calculations for light supersymmetric particle scattering off light nuclei," Physical Review C, vol. 61, no. 5, pp. 546121$5461212,2000$.

[28] E. Kolbe and K. Langanke, "Role of $\mathcal{v}$-induced reactions on lead and iron in neutrino detectors," Physical Review C, vol. 63, no. 2, Article ID 025802, 11 pages, 2001.

[29] N. Jachowicz, K. Heyde, and S. Rombouts, "Many-body description of neutrino-nucleus interactions," Nuclear Physics A, vol. 688, no. 1-2, pp. 593-595, 2001.

[30] P. C. Divari, "Cross-sections for neutral-current neutrino scattering off ${ }^{82}$ Se isotope," Acta Physica Polonica B, vol. 41, no. 6, pp. 1339-1353, 2010.

[31] P. C. Divari, V. C. Chasioti, and T. S. Kosmas, "Neutral current neutrino- ${ }^{98}$ Mo reaction cross sections at low and intermediate energies," Physica Scripta, vol. 82, no. 6, Article ID 065201, 2010.

[32] V. Tsakstara, T. S. Kosmas, and P. C. Divari, "Investigating the nuclear response of Te isotopes to SN-neutrinos," Journal of Physics: Conference Series, vol. 203, Article ID 012093, 2010.

[33] K. G. Balasi, T. S. Kosmas, and P. C. Divari, "Inelastic neutrino scattering off stable even-even Mo isotopes at low and intermediate energies," Progress in Particle and Nuclear Physics, vol. 64, no. 2, pp. 414-416, 2010.

[34] V. C. Chasioti, T. S. Kosmas, and P. C. Divari, "Inelastic neutrino-nucleus reaction cross sections at low neutrino-energies," Progress in Particle and Nuclear Physics, vol. 59, no. 1, pp. 481-485, 2007.

[35] H. A. Bethe, "Supernova mechanisms," Reviews of Modern Physics, vol. 62, no. 4, pp. 801-866, 1990.

[36] H. T. Janka, K. Langanke, A. Marek, G. Martínez-Pinedo, and B. Müller, "Theory of core-collapse supernovae," Physics Reports, vol. 442, no. 1-6, pp. 38-74, 2007.

[37] A. Mezzacappa, "Ascertaining the core collapse supernova mechanism: the state of the art and the road ahead," Annual Review of Nuclear and Particle Science, vol. 55, pp. 467-515, 2005.

[38] J. F. Beacom, W. M. Farr, and P. Vogel, "Detection of supernova neutrinos by neutrino-proton elastic scattering," Physical Review D, vol. 66, no. 3, Article ID 033001, 2002.

[39] K. Takahashi, M. Watanabe, K. Sato, and T. Totani, "Effects of neutrino oscillation on the supernova neutrino spectrum," Physical Review D, vol. 64, no. 9, Article ID 093004, 2001.

[40] T. Totani, K. Sato, H. E. Dalhed, and J. R. Wilson, "Future detection of supernova neutrino burst and explosion mechanism," Astrophysical Journal Letters, vol. 496, no. 1, pp. 216-225, 1998.

[41] M. T. Keil, G. G. Raffelt, and H. T. Janka, "Monte Carlo study of supernova neutrino spectra formation," Astrophysical Journal Letters, vol. 590, no. 2, pp. 971-991, 2003.

[42] I. Gill-Botella and A. Rubbia, "Oscillation effects on supernova neutrino rates and spectra and detection of the shock breakout in a liquid argon TPC," Journal of Cosmology and Astroparticle Physics, vol. 10, article 009, 2003.

[43] M. S. Athar, S. Ahmad, and S. K. Singh, "Supernova neutrino induced inclusive reactions on ${ }^{56} \mathrm{Fe}$ in terrestrial detectors," Physical Review C, vol. 71, no. 4, Article ID 045501, 7 pages, 2005.

[44] M. S. Athar, S. Ahmad, and S. K. Singh, "Neutrino nucleus cross sections for low energy neutrinos at SNS facilities," Nuclear Physics A, vol. 764, no. 1-4, pp. 551-568, 2006.

[45] A. Meucci, C. Giusti, and F. D. Pacati, "Neutral-current neutrino-nucleus quasielastic scattering," Nuclear Physics A, vol. 744, pp. 307-322, 2004. 
[46] E. Kolbe, K. Langanke, and P. Vogel, "Estimates of weak and electromagnetic nuclear decay signatures for neutrino reactions in Super-Kamiokande," Physical Review D, vol. 66, no. 1, Article ID 013007, 5 pages, 2002.

[47] T. W. Donnelly and J. D. Walecka, "Elastic magnetic electron scattering and nuclear moments," Nuclear Physics, Section A, vol. 201, no. 1, pp. 81-106, 1973.

[48] P. Ring and P. Schuck, The Nuclear Many-Body Problem, Springer, New York, NY, USA, 1980.

[49] A. Bohr, B. R. Mottelson, and D. Pines, "Possible Analogy between the excitation spectra of nuclei and those of the superconducting metallic state," Physical Review, vol. 110, no. 4, pp. 936-938, 1958.

[50] A. Bohr and B. R. Mottelson, Nuclear Structure, vol. 1, Benjamin, New York, NY, USA, 1969.

[51] K. Holinde, "Two-nucleon forces and nuclear matter," Physics Reports, vol. 68, no. 3, pp. 121-188, 1981.

[52] W. A. Kamiński and A. Faessler, "Description of the ground-state pionic double charge exchange reaction on ${ }^{128,130}$ Te," Nuclear Physics, Section A, vol. 529, no. 4, pp. 605-632, 1991.

[53] G. Audi, A. H. Wapstra, and C. Thibault, "The Ame2003 atomic mass evaluation-(II). Tables, graphs and references," Nuclear Physics A, vol. 729, no. 1, pp. 337-676, 2003.

[54] Y. Giomataris and J. D. Vergados, "A network of neutral current spherical TPCs for dedicated supernova detection," Physics Letters, Section B, vol. 634, no. 1, pp. 23-29, 2006.

[55] C. J. Horowitz, K. J. Coakley, and D. N. McKinsey, "Supernova observation via neutrino-nucleus elastic scattering in the CLEAN detector," Physical Review D, vol. 68, no. 2, Article ID 023005, 2003.

[56] J. Engel, "Nuclear form factors for the scattering of weakly interacting massive particles," Physics Letters, Section B, vol. 264, no. 1-2, pp. 114-119, 1991.

[57] E. A. Paschos and A. Kartavtsev, "Coherent pion production on nuclei," Nuclear Physics B, vol. 159, no. 1, pp. 203-208, 2006.

[58] G. Rai, A. Arthur, F. Bieser et al., "A TPC detector for the study of high multiplicity heavy ion collisions," IEEE Transactions on Nuclear Science, vol. 37, no. 2, pp. 56-64, 1990.

[59] I. Giomataris, I. Irastorza, I. Savvidis et al., "A novel large-volume spherical cetector with proportional amplification read-out," Journal of Instrumentation, vol. 3, Article ID JINST 3 P09007, 2008.

[60] S. Aune, P. Colas, H. Deschamps et al., "Progress on a spherical TPC for low energy neutrino detection," Journal of Physics: Conference Series, vol. 39, p. 281, 2006.

[61] S. Aune, P. Colas, J. Dolbeau et al., "NOSTOS: a spherical TPC to detect low energy neutrinos," AIP Conference Proceedings, vol. 785, pp. 110-118, 2005.

[62] S. Andriamonje, S. Aune, E. Bougamont et al., "Spherical TPC development and trends," Journal of Physics: Conference Series, vol. 179, Article ID 012003, 2009.

[63] Y. Giomataris and J. D. Vergados, "Neutrino properties studied with a triton source and a large spherical TPC," Nuclear Instruments and Methods in Physics Research, Section A, vol. 530, no. 3, pp. 330-358, 2004.

[64] I. Giomataris, S. Aune, P. Colas et al., "NOSTOS experiment and new trends in rare event detection," Nuclear Physics B, vol. 150, no. 1-3, pp. 208-213, 2006.

[65] J. D. Vergados, F. T. Avignone, and I. Giomataris, "Coherent neutral current neutrino-nucleus scattering at a spallation source: a valuable experimental probe," Physical Review D, vol. 79, no. 11, Article ID 113001, 2009.

[66] I. Giomataris and J. D. Vergados, "Supernova detection via a network of neutral current spherical TPC's," AIP Conference Proceedings, vol. 847, pp. 140-146, 2006. 

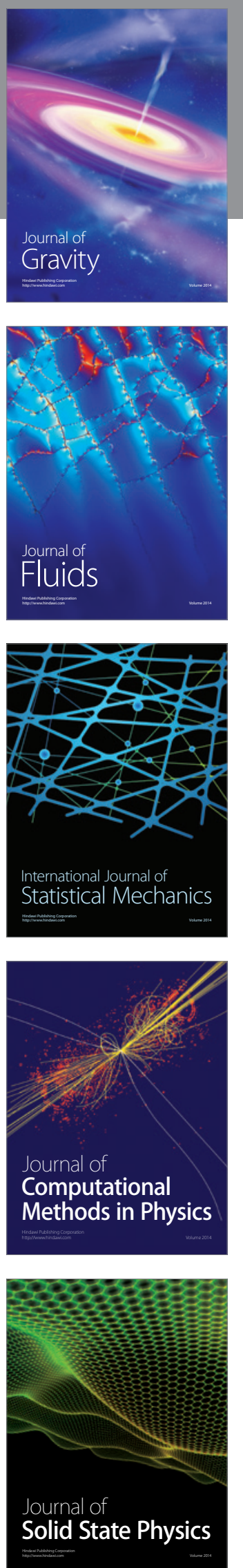

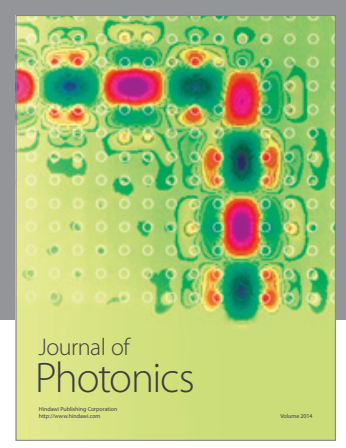

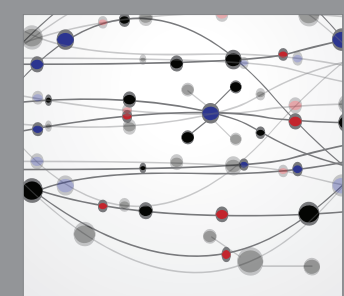

The Scientific World Journal
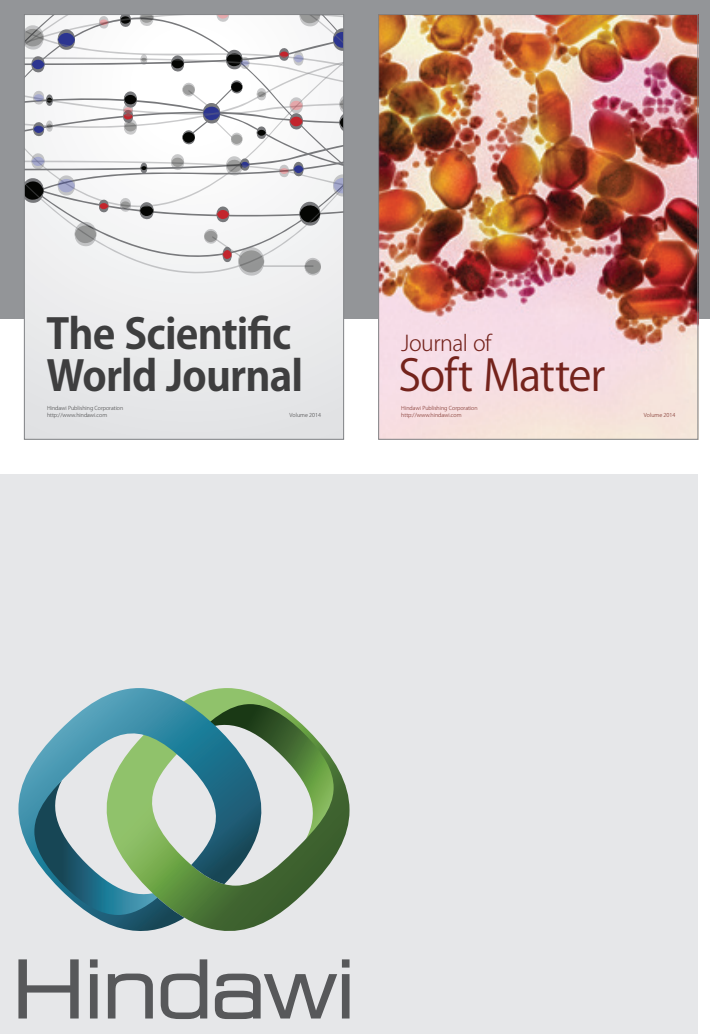

Submit your manuscripts at

http://www.hindawi.com
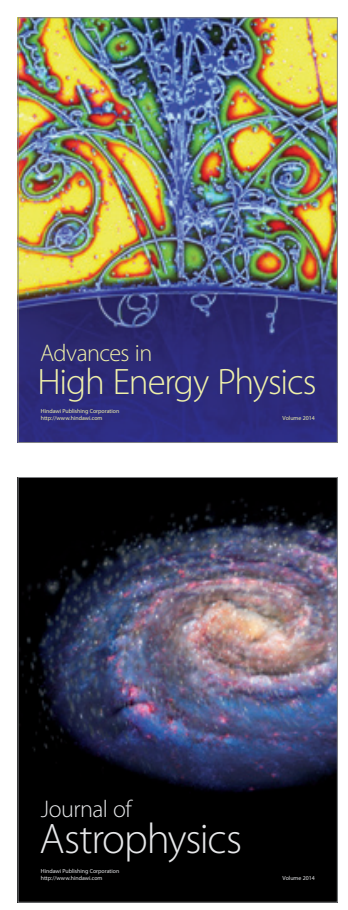
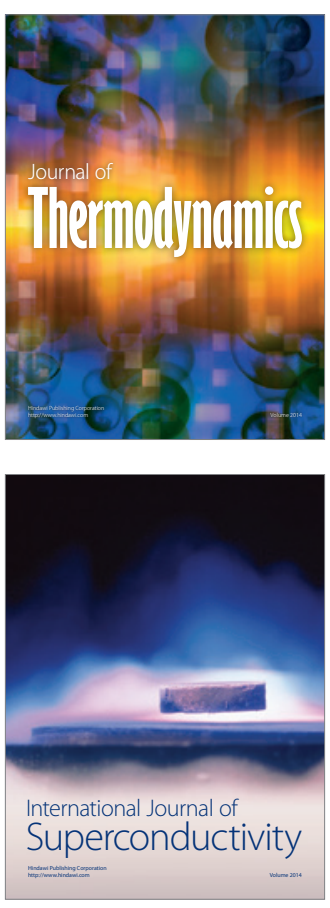
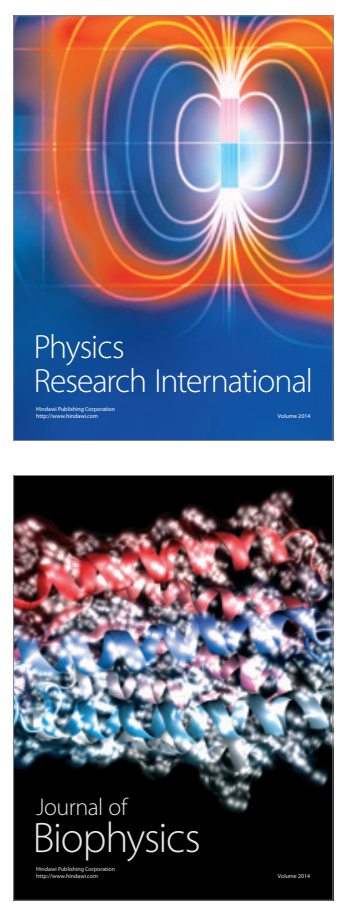
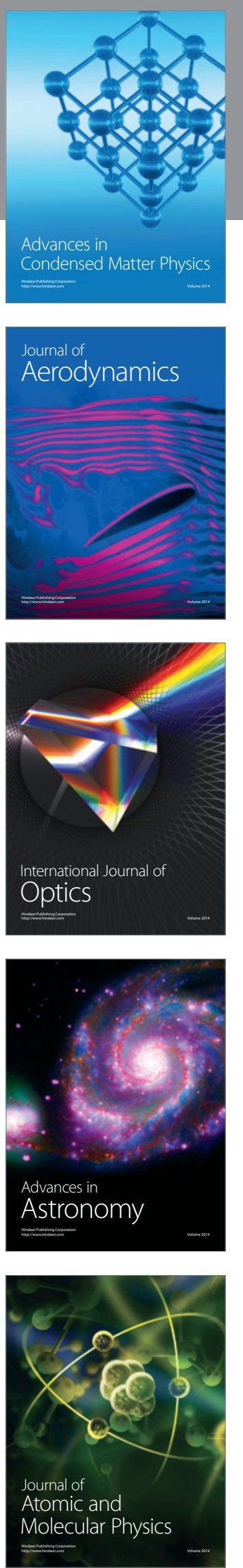